\title{
[gw22-e1011] EFFECT OF TRANSPLANTATION WITH MESENCHYMAL STEM CELLS ON ACUTE MYOCARDIAL INFARCTION IN SWINE
}

Peng Chaoquan, ${ }^{1}$ Yang Ke, ${ }^{1}$ Xiang Peng, ${ }^{2}$ Zhang Chengxi, ${ }^{1}$ Zou Liyuan, ${ }^{1}$ JingFeng Wang ${ }^{1}{ }^{1}$ Department of Cardiology, Third Affiliated Hospital of Sun Yat-sen, Guangdong, China; ${ }^{2}$ Zhongshan University Stem Cell and Tissue Engineering Research Center, China

\subsection{6/heartjnl-2011-300867.419}

Objective To investigate the effects of treatment with autologous MSCs by transplantation on AMI in swine.

Method MSCs were injected by OTW balloon in the experimental group $(n=10)$, while normal saline was injected into the control $(n=10)$. Improvements in cardiac function were evaluated by UCG and SPECT.

Result Eight weeks after transplantation, the LVEF and FS were significantly increased in the MSCs group as compared with pretransplantation $(p<0.05)$, with a significant decrease in EDV and ESV ( $\mathrm{p}<0.05)$. However, no significant differences were observed in the saline group. The LVEF and FS were significantly increased in the MSC group as compared with the saline group $(p<0.05)$, with a significant decrease in EDV and ESV $(p<0.05)$. SPECT revealed that the myocardial filling defect was reduced and the amount of surviving myocardium was significantly increased.

Conclusion Intracoronary transplantation of MSCs can increase cardiac function and promote myocardial viability. 